\section{S4D:6 SLE COMPRISES FOUR IMMUNE-PHENOTYPES, WHICH DIFFER REGARDING HLA-DRB1 AND CLINICAL ASSOCIATIONS}

${ }^{1} \mathrm{LM}$ Diaz Gallo, ${ }^{1} \mathrm{E}$ Lundström, ${ }^{1} \mathrm{~V}$ Oke, ${ }^{2} \mathrm{~K}$ Elvin, ${ }^{3} \mathrm{YL}$ Wu, ${ }^{1} \mathrm{~J}$ Gustafsson, ${ }^{4} \mathrm{~A}$ Jönsen, ${ }^{5} \mathrm{D}$ Leonard, ${ }^{1} \mathrm{~A}$ Zickert, ${ }^{5} \mathrm{G}$ Nordmark, ${ }^{4} \mathrm{AA}$ Bengtsson, ${ }^{5}$ J Sandling, ${ }^{5} \mathrm{~L}$ Rönnblom, ${ }^{1}$ I Gunnarsson, ${ }^{3} \mathrm{CY}$ Yu, ${ }^{1} \mathrm{~L}$ Padyukov, ${ }^{1} \mathrm{E}$ Svenungsson. ${ }^{1}$ Department of Medicine, Rheumatology Unit, Karolinska Institutet/Karolinska University Hospital, Stockholm, Sweden; ${ }^{2}$ Department of Clinical Immunology and Transfusion Medicine, Unit of Clinical Immunology, Karolinska Institutet/Karolinsk, Stockholm, Sweden; ${ }^{3}$ The Research Institute at Nationwide Childrens Hospital, Columbus, Ohio, USA; ${ }^{4}$ Department of Clinical Sciences, Section of Rheumatology, Lund University, Skåne University Hospital, Lund, Sweden; ${ }^{5}$ Department of Medical Sciences, Section of Rheumatology, Uppsala University, Uppsala, Sweden

\subsection{6/lupus-2018-abstract.25}

SLE is a heterogeneous disease including diverging clinical symptoms, autoantibodies and genetic susceptibility. Hitherto unrecognised patterns may define sub-phenotypes with different pathogenesis and specific treatment needs. Based on autoantibody profile we therefore investigated phenotypic clusters and explored cluster associations with clinical manifestations and one of the most important genetic risk factors for SLE, HLA-DRB1 alleles.

908 SLE Caucasian patients and 3654 age- gender- and ethnicity-matched healthy controls (HC) were included. We determined the occurrence of 13 autoantibodies: dsDNA, nucleosomes, ribosomal P, RNP68, RNPA, Sm, Sm/RNP, SSA52, SSA60, SSB, aCL-IgG/IgM and aB2GP1. HLA-DRB1 typing was performed by sequence-specific primer polymerase chain reaction assay. Cluster analysis was done using Gower distance matrix, followed by partition around medoids cluster calculation and Silhouette metric for number of clusters validation. Chi-square test, odds ratios (OR), 95\% confidence intervals and false discovery rate $\mathrm{p}$ value $(\mathrm{p})$ were calculated for the association tests.

Four clusters were defined based on autoantibody occurrence.

1. $29 \%$, dominated by anti-SSA52/60/SSB positivity is strongly associated with HLA-DRB1*03 when compared to HC (4.1 [3.4-4.9] $\mathrm{p}=6.4 \mathrm{E}-56)$ and other clusters (OCs) (2.9[93.33.6] $\mathrm{p}=1.1 \mathrm{E}-19)$. Discoid lesions were more common vs OCs $(1.8[1.3-2.6] \mathrm{p}=0.02)$.

2. $29 \%$, dominated by anti-SmRNP/Sm/DNA/RNPA/RNP68/ nucleosome, was specifically associated with HLA-DRB1*15 when compared to HC (1.7[1.6-2.1] $\mathrm{p}=5.7 \mathrm{E}-6)$ and other clusters $(1.5[1.1-1.9] \mathrm{p}=0.01)$. Nephritis was common vs OCs $(1.9[1.4-2.7) \mathrm{p}=2 . \mathrm{E}-03)$

3. $24 \%$, dominated by anti-B2GP1/aCL-IgG/IgM, was associated with HLA-DRB1*04 when compared with other clusters (1.8[1.4-2.4] $\mathrm{p}=2 \mathrm{E}-4)$. More thrombotic events vs OCs were observed in this group $(1.84[1.3-2.6] \mathrm{p}=0.01)$

4. $18 \%$ was negative for the 13 tested autoantibodies and was not associated with any specific HLA-DRB1 alleles and it was not associated as risk factor for any of the evaluated clinical manifestations.

We demonstrate that immune-phenotypes/clusters in SLE can fit into a frame of HLA-DRB1 alleles and that the overall association between SLE and HLA-DRB1*03 and HLADRB1*15 seems to be driven mainly by clusters 1 and 2, respectively. We also confirm previous observations that auto- antibody clusters associate with clinical symptoms. We believe that these results could be used to redefine SLE, determine predictive biomarkers and inclusion criteria for clinical trials.

\section{S4D:7 NEXT GENERATION SEQUENCING IN HEMATOPOIETIC PROGENITORS OF MURINE SLE MODEL REVEALS ABERRANT REGULATION OF CEBPIA EXPRESSION}

${ }^{1} \mathrm{M}$ Grigoriou, ${ }^{1} \mathrm{P}$ Verginis, ${ }^{2} \mathrm{C}$ Nikolaou, ${ }^{3} \mathrm{P}$ Pavlidis, ${ }^{4} \mathrm{E}$ Dermitzakis, ${ }^{5} \mathrm{G}$ Bertsias, ${ }^{1,6} \mathrm{D}$ Boumpas, ${ }^{1} \mathrm{~A}$ Banos. 'Biomedical Research Foundation of the Academy of Athens, Greece; ${ }^{2}$ Department of Biology, University of Crete, Heraklion, Crete, Greece; ${ }^{3}$ Institute of Molecular Biology and Biotechnology-FORTH, Heraklion, Crete, Greece; ${ }^{4}$ Department of Genetic Medicine and Development, Medical School, University of Geneva, Geneva, Switzerland; ${ }^{5}$ Medical School, University of Crete, Heraklion, Crete, Greece; ${ }^{6}$ Joint Academic Rheumatology Program, Medical School, National and Kapodistrian University of Athens, Greece

\subsection{6/lupus-2018-abstract.26}

Background and purpose All blood cell lineages that have implicated to the pathogenesis of SLE originate from the Hematopoietic Stem Cells (HSCs). Studying HSCs may help to dissect fundamental immune aberrations in SLE and elucidate the HSC contribution to the pathogenesis of the disease. Materials and methods HSCs were isolated from either healthy C57/BL6 or NZBxNZW/F1 lupus-prone mice bone marrow. The selection markers used are Lin-Sca-1+c-Kit+for LSK compartment that encompasses both long- and short-term HSCs as well as multipotent progenitors (MPP). Flow cytometry cell sorting of LSK was used for enumeration, RNA extraction, qPCR and cell cultures. Paired-end RNA-sequencing analysis was performed by the Illumina HiSeq 2000 platform.

Results We found significantly increased numbers of LSK in the $\mathrm{BM}$ of lupus NZBxNZW/F1 mice with established disease as compared to pre-diseased NZBxNZW/F1 mice in combination with evidence of them exiting the latent state and progression of cell cycle and aberrant differentiation with skewing towards the myeloid lineage. Transcriptome analysis revealed 800 differentially expressed genes (DEGs) (FC>1.5, q<0.05) in diseased lupus mice compared to pre-diseased with enrichment in transcription factors involved in hematopoiesis, regulation of immune responses and HSC function/homeostasis. We selected Cebp $\alpha$ (FC -0,88) -a master regulator of myeloid differentiation, self-renewal and resistance to stress-induced apoptosis of HSCs- for further investigation. qPCR analysis showed decreased Cebp $\alpha$ expression during the progression of the disease in SLE but increased Cebp $\alpha$ in aged healthy C57/ BL6 mice. In vitro stimulation with IFN $\alpha$ decreased Cebp $\alpha$ expression in lupus -but not in healthy LSK. Serum from prediseased NZBxNZW/F1 decreased Cebp $\alpha$ expression only in pre-diseased LSK. Experiments to reverse Cebp $\alpha$ downregulation (using lenti-virus and modifiers of the metabolomics) are in progress.

Conclusions HSC RNA-seq analysis suggests both intrinsic and extrinsic influences resulting in downregulation of Cebp $\alpha$ in murine lupus. SLE HSCs have pronounced expansion, enhanced proliferation and aberrant differentiation -in part due to the effects of IFN $\alpha$. Together these results suggest a decreased capacity of lupus HSCs to respond to stressors which may account for the cytopenias and the infections in SLE. 


\section{S5a - Biomarker \& indices}

\section{S5A:4 CIRCULATING TYPE I, II AND III INTERFERONS (IFNS) ASSOCIATE WITH IFN-SCORES, BUT DEFINE DISTINCT SUBSETS OF ACTIVE SLE}

${ }^{1} V$ Oke, ${ }^{1} I$ Gunnarsson, ${ }^{2} \mathrm{~J}$ Dorschner, ${ }^{1} \mathrm{~A}$ Zickert, ${ }^{3} \mathrm{~TB}$ Niewold, ${ }^{1} \mathrm{E}$ Svenungsson. ${ }^{1}$ Rheumatology Clinic, Department of Medicine, Karolinska Institutet, Karolinska University Hospital, Stockholm, Sweden; '2Division of Rheumatology and Department of Immunology, Mayo Clinic, Rochester, MN, USA; ${ }^{3}$ New York University School of Medicine, New York, NY, USA

\subsection{6/lupus-2018-abstract.27}

Background Serum induced IFN gene expression (IFN-score) is considered a golden standard to assess IFN activity in SLE. So far, IFN-scores have not been compared to serum levels of type I, II and III IFNs.

Aim To investigate how IFN-scores and SLE manifestations relate to serum levels of IFNs type I (alphas), II (gamma) and III (lambda 1).

Methods 461 SLE patients and 322 controls were included. IFN-score was measured by WISH cell assay. INF-alphas and IFN-lambda1 were measured by ELISA. IFN-gamma was measured by MSD 30-plex assay.

Results SLE patients had higher IFN-scores and higher levels of IFN-alphas, IFN-gamma and IFN-lambda1 $(\mathrm{p}<0.001)$. IFNscores correlated with levels of IFN-gamma and IFN-alpha $(\mathrm{rho}=0.39$, and $\mathrm{rho}=0.25, \mathrm{p}<0.0001)$. Further, patients were grouped according to high levels ( $>3$ rd quartile) of each IFN/IFN-score. The group with high IFN-scores had higher disease activity (SLAM, SLEDAI): weight loss (41\%), fatigue (33\%), fever (39\%), rash (44\%), lymphadenopathy (45\%), arthritis $(40 \%)$, nephritis $(55 \%)(\mathrm{p}<0.01)$. Interestingly, incidence of neuropsychiatric SLE, antiphospholipid (aPL) antibodies (abs), and also damage score was lower $(p<0.05)$.

The characteristics of IFN-gamma high group included higher disease activity (SLAM, SLEDAI), and specifically: active nephritis (52\%), lymphadenopathy (40\%), arthritis (42\%), lymphopenia $(37 \%)$, anaemia $(35 \%)$ and positivity for Sm $(41 \%)$, SmRNP (36\%) and RNP68 (45\%), Ro52 (35\%) and Ro60 $(33 \%)(\mathrm{p}<0.03)$.

The common features of IFN-alpha high group included younger age, shorter disease duration, active rash (34\%), lymphadenopathy (43\%), Ro52 (38\%) and La (43\%) $(\mathrm{p}=0.01)$. Presence of aPL abs and previous vascular events were lower and renal affection was uncommon $(\mathrm{p}<0.01)$.

In general, high IFN-scores reflected SLE manifestations that could be further stratified by high IFN-gamma levels and to a lesser extent by high IFN-alpha. High IFN-lambda1 did not define any phenotype of active SLE, except presence of anti-nucleosome abs.

Conclusion We demonstrate that high IFN-score associate more strongly with type II rather than type I IFNs. Importantly, major manifestations of SLE: active nephritis and arthritis, and also anti-Sm/SmRNP antibodies associate with IFNgamma; while rash associate with IFN-alpha.

Our findings are of major importance while tailoring clinical trials with anti-IFN therapies and demonstrate that importance of IFN-gamma has so far been underscored.

\section{S5A:5 GALECTIN-9 IS PRODUCED BY DENDRITIC CELLS AND SERUM LEVELS OUTPERFORM CXCL10 AS A BIOMARKER TO DETECT THE IFN SIGNATURE IN SLE AND APS}

${ }^{1} \mathrm{LL}$ van den Hoogen, ${ }^{1} \mathrm{JAG}$ van Roon, ${ }^{1} \mathrm{~J}$ Wienke, ${ }^{1} \mathrm{~F}$ van Wijk, ${ }^{2} \mathrm{RDE}$ Fritsch-Stork, ${ }^{1}$ TR Radstake. 'Laboratory of Translational Immunology/Rheumatology and Clinical Immunology, University Medical Centre Utrecht, Utrecht, The Netherlands; ${ }^{2} 1 s t$ Medical Department, Hanusch Hospital of WGKK, Ludwig Boltzmann Institute of Osteology, Hanusch Hospital, Vienna, Austria

\subsection{6/lupus-2018-abstract.28}

Objective The most prominent alteration in the immune system of patients with systemic lupus erythematosus (SLE) and antiphospholipid syndrome (APS) is the increased expression of type I interferon (IFN) inducible genes, known as the IFN signature. This signature is related to disease activity, vascular disease and predicts future flares and the response to anifrolumab. There is an urgent need for easily measurable biomarkers to detect an IFN signature. Galectin-9 may steer Tcell responses and is a potential biomarker for autoimmune diseases. In the present study we investigated the performance of galectin-9 as a biomarker to detect the IFN signature in SLE and APS and its production by dendritic cell (DC) subsets.

Methods Serum levels of galectin-9 and CXCL10 were measured by Luminex in an identification cohort $(n=43)$ and replication cohort $(n=148)$ of patients with SLE, SLE +APS and primary APS (PAPS) and healthy controls (HC). Isolated monocytes were used to quantify IFN scores by measuring the expression of 4 type I IFN inducible genes. The performance of potential biomarkers for the IFN signature were assessed by Receiver Operating Characteristics curves. RNA sequencing on plasmacytoid and myeloid DC isolated from patients with SLE, SLE +APS and PAPS $(n=54)$ were analysed to identify potential sources of galectin-9 in SLE and APS.

Results In both identification and replication cohort, serum levels of galectin-9 and CXCL10 were elevated in SLE, SLE + APS and PAPS patients as compared with HC (all $\mathrm{p}<0.05)$ and both galectin-9 and CXCL10 correlated with the

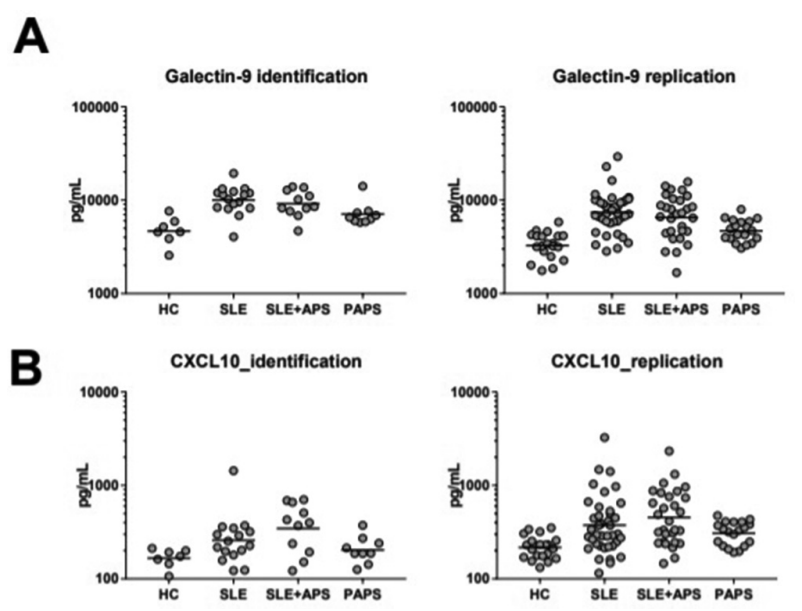

Abstract S5A:5 Figure 1 